\section{Sobrevida de pacientes em diálise no SUS no Brasil}

\author{
Survival analysis of dialysis patients in the \\ Brazilian Unified National Health System
}

\section{Abstract}

1 Faculdade Medicina, Universidade Federal de Minas Gerais, Belo Horizonte, Brasil.

2 Faculdade de Farmácia, Universidade Federal de Minas Gerais, Belo Horizonte, Brasil.

Correspondência D. A. C. Szuster Faculdade Medicina, Universidade Federal de Minas Gerais.

Av. Alfredo Balena 190, Belo Horizonte, MG 30190-170, Brasil.

danieleacampos@gmail.com
The aim of this study was to analyze the survival of patients who initiated renal replacement therapy (RRT) with hemodialysis or peritoneal dialysis in the Brazilian Unified National Health System from 2002 to 2004. This was an observational, prospective, non-concurrent study. The study used the National Database for Renal Replacement Therapies resulting from probabilistic matching of Authorization of High-Complexity Procedures/Outpatient Information System and the Mortality Information System. The study included patients admitted in 2002 and 2003, with 3 months of treatment, and 18 years or older. Of the 31,298 patients, the majority: began RRT with hemodialysis, were male, with mean age 54 years, and living in the Southeast region and in municipalities with a mean HDI of 0.78. Increased risk of death was associated with: female gender, age greater than 55 years, diagnosis of diabetes mellitus, peritoneal dialysis, and not residing in the Southeast region. Residing in cities with higher HDI was associated with lower risk. Adjusted risk was HR $=1.17$ in favor of hemodialysis. The results suggest shorter survival for peritoneal dialysis and older patients. It is thus necessary to support policies to better evaluate the RRT modality with studies that further elucidate the findings.

Chronic Renal Insufficiency; Dialysis; Survivorship
Daniele Araújo Campos Szuster 1

Waleska Teixeira Caiaffa 1

Eli Iola Gurgel Andrade 1

Francisco de Assis Acurcio 2

Mariangela Leal Cherchiglia 1

\section{Introdução}

O número de pacientes com insuficiência renal crônica terminal tratados com terapias renais substitutivas cresce no mundo a uma taxa anual de, aproximadamente, $7 \%$ ao ano. Esta taxa excede a taxa de crescimento da população 1 . As diálises, em suas duas formas - hemodiálise ou diálise peritoneal, são as modalidades de tratamento mais frequentes. No Brasil, entre $2000 \mathrm{e}$ 2006, o crescimento do número de pacientes em diálise foi cerca de $9 \%$ ao ano, sendo o Sistema Único de Saúde (SUS) responsável por 89\% do financiamento desse tratamento ${ }^{2}$.

Vários fatores influenciam a escolha da modalidade de diálise, incluindo as características individuais e clínicas do paciente no início do tratamento, as preferências dos pacientes e médicos e a localização geográfica 3,4,5. Alguns estudos também têm enfocado a importância de fatores econômicos na escolha da modalidade tais como a forma de pagamento do tratamento e a disponibilidade de recursos 6 . Segundo Just et al. 7, nos lugares onde há poucos incentivos financeiros para o prestador de serviço quando a diálise peritoneal é escolhida, esta é pouco utilizada. O uso da diálise peritoneal também sofre impacto da empregabilidade médica. Os resultados dos tratamentos, medidos em termos de sobrevida, morbidade e qualidade de vida, são também considerados na escolha da 
modalidade de diálise. A hemodiálise é o tratamento mais realizado no Brasil para tratamento da insuficiência renal crônica terminal, numa proporção de $90 \%$ das diálises 2 . É sabido que a alocação entre as modalidades de diálise não é feita randomicamente, sendo os pacientes mais velhos e diabéticos encaminhados para a diálise peritoneal, o que poderia impactar o risco de morte para esta modalidade 3 .

$\mathrm{Na}$ última década, vários estudos foram conduzidos investigando a sobrevida entre pacientes em hemodiálise e em diálise peritoneal 5,8,9,10,11,12,13,14,15,16. No entanto, os resultados são controversos, ocasionados, muitas vezes, por diferenças metodológicas ou na construção dos grupos populacionais estudados 4,9,17.

No Brasil é insuficiente o número de estudos que avaliam, em âmbito nacional, a sobrevida dos pacientes em ambas as modalidades de diálise 18 . O objetivo deste trabalho foi realizar uma análise da sobrevida entre os pacientes que iniciaram o tratamento em hemodiálise e em diálise peritoneal no SUS, no período de janeiro de 2002 a dezembro de 2003.

\section{Método}

Trata-se de um estudo do tipo observacional, prospectivo não concorrente, derivado de estudo anterior intitulado Projeto TRS - Avaliação Econômico-Epidemiológica das Terapias Renais Substitutivas no Brasil, conduzido pelo Grupo de Pesquisa em Economia da Saúde da Universidade Federal de Minas Gerais (GPES/UFMG) 19. A fonte de dados utilizada foi a Base Nacional em Terapias Renais Substitutivas, desenvolvida por meio da técnica de relacionamento determinístico-probabilístico entre os bancos de dados de Autorização de Procedimentos de Alta Complexidade/Custo, do Sistema de Informações Ambulatoriais (SIA) do SUS, e do Sistema de Informações sobre Mortalidade (SIM), com o objetivo de habilitar o seguimento de coorte 19,20 . A população estudada incluiu todos pacientes incidentes registrados na Base Nacional em Terapias Renais Substitutivas que iniciaram diálise entre 1o de janeiro de 2002 a 31 de dezembro de 2003 ( $\mathrm{N}=$ 37.350). Foram excluídos os pacientes menores de 18 anos e maiores de 100 anos $(n=1.316)$ e os que foram a óbito nos três primeiros meses de tratamento (4.736). Todos foram acompanhados até 31 de dezembro de 2004.

A variável dependente foi o tempo (em meses) decorrido entre a data de entrada na modalidade inicial de tratamento, até data do óbito do paciente. Foi definida como modalidade inicial de tratamento (hemodiálise ou diálise peritone- al) a primeira na qual o paciente permaneceu por pelo menos três meses consecutivos, não tendo sido consideradas as mudanças posteriores de modalidade.

As variáveis independentes foram: sexo, idade à entrada em terapia renal substitutiva - estratificada entre idade inferior e superior a 55 anos; região de residência no primeiro registro; causa básica de insuficiência renal crônica terminal no primeiro registro (10a revisão de Classificação Internacional de Doenças - CID-10) e o Índice de Desenvolvimento Humano (IDH) do ano de 2000, do Programa das Nações Unidas para o Desenvolvimento (PNUD) como estimador das condições socioeconômicas dos municípios de residência dos pacientes. O IDH é indicador composto que engloba três dimensões: renda, educação e esperança média de vida 21 .

Foi realizada análise descritiva por meio de distribuições de frequências, medidas de tendência central e de variabilidade para as características estudadas. O teste $\chi^{2}$ de Pearson foi utilizado para verificar diferenças de proporções entre variáveis categóricas e o teste $\mathrm{t}$ de Student para comparação de variáveis contínuas. Para a variável IDH, pelas características não paramétricas foi realizado teste de Mann-Whitney.

Para as análises de sobrevida os pacientes foram identificados de acordo com sua modalidade inicial de terapias renais substitutivas. Primeiro analisou-se a sobrevida como preconizado por van Biesen et al. 16: (i) sobrevida em primeira modalidade; (ii) sobrevida "intention-to-treat", considerando somente o tempo em diálise; e (iii) sobrevida total, considerando o tempo total em tratamento, incluindo o tempo em diálises e transplante renal. Neste caso, o evento final considerado foi o óbito e os pacientes foram censurados por perda de seguimento ou ao final do período de estudo.

Como na análise preliminar, observou-se pequena variação no tempo médio de sobrevida nas diferentes abordagens propostas por van Biesen et al. 16, optou-se, neste artigo, por realizar as análises utilizando a sobrevida total, considerando o tempo total em terapias renais substitutivas.

Para estimar a probabilidade acumulada do tempo de sobrevida nos participantes aplicou-se o método não paramétrico de Kaplan-Meier. A comparação das diferentes curvas para os subgrupos correspondentes às variáveis de exposição foi baseada no teste de log-rank.

Para a identificação do efeito independente das variáveis explicativas, utilizou-se o modelo multivariado de riscos proporcionais de Cox (harzard ratio - HR). A premissa de risco relativo constante no tempo foi avaliada por meio 
da análise do gráfico de log minus log, para cada variável explicativa. A mesma estimativa foi usada para avaliar o HR entre as modalidades hemodiálise e diálise peritoneal de acordo com as características: sexo, idade à entrada em terapias renais substitutivas e presença ou não de diabetes mellitus como causa básica da insuficiência renal crônica terminal. As análises foram realizadas no software SPSS versão 16 (SPSS Inc., Chicago, Estados Unidos), sendo adotado um nível de $5 \%$ de significância.

O projeto terapia renal substitutiva foi aprovado pelo Comitê de Ética em Pesquisa da Universidade Federal de Minas Gerais, parecer ETIC $397 / 2004$.

\section{Resultados}

Nos anos de 2002 e 2003, 31.298 pacientes elegíveis para o estudo iniciaram diálises no Brasil. A distribuição dos pacientes estudados obedeceu ao padrão dos 90.356 pacientes incidentes constantes na Base Nacional em Terapias Renais Substitutivas de acordo com as variáveis região de residência no primeiro registro, sexo, idade à entrada em terapias renais substitutivas e modalidade ao início do tratamento. A distribuição foi proporcionalmente diferente em relação aos óbitos, uma vez que foram excluídos óbitos ocorridos nos três meses iniciais, como preconizado pela literatura 5 .

A maioria dos pacientes $(90 \%)$ iniciou o tratamento renal substitutivo em hemodiálise, era do sexo masculino, com idade média de 54 anos e residente na Região Sudeste; o IDH médio para as cidades de residência dos pacientes foi de 0,78 . O principal diagnóstico de insuficiência renal crônica terminal foi hipertensão, seguido por doenças cardiovasculares. No entanto, um grande número de pacientes apresentava diagnóstico indeterminado, dificultando a identificação etiológica. A grande maioria dos pacientes permaneceu na mesma modalidade inicial de tratamento durante todo o período de estudo e $34 \%$ dos pacientes evoluíram para o óbito (Tabela 1).

Para o grupo com início de tratamento em hemodiálise a maioria era do sexo masculino com idade média de 53 anos, residente nas regiões Sudeste e Nordeste. O grupo que iniciou em diálise peritoneal era na maioria do sexo feminino, apresentava idade média mais elevada (59,5 anos), residia na Região Sudeste e em municípios com maior índice de IDH. Dos pacientes que iniciaram em hemodiálise $6 \%$ foram transplantados e $4 \%$ transitaram para diálise peritoneal, enquanto entre os pacientes que iniciaram em diálise peritoneal, $4 \%$ foram transplantados e $15 \%$ transitaram para hemodiálise durante o período de seguimento.

Ao final do período de seguimento, $42 \%$ dos pacientes em diálise peritoneal e $33 \%$ em hemodiálise foram a óbito, relacionados principalmente com a diabetes mellitus e doenças cardiovasculares.

Os tempos médios de sobrevida para o 1ํ, 2으 e 3o anos de tratamento foram, respectivamente, de 10,7, 19,3 e 26,5 meses para os iniciantes em hemodiálise sendo estatisticamente diferente dos iniciantes em diálise peritoneal, cujos tempos médios foram: 10,7, 18,5 e 24,2 meses. As funções de sobrevida para o 1o, 2o e $3 \underline{o}$ anos de tratamento dos iniciantes em hemodiálise foram, respectivamente, de 79\%, 69\% e 66,5\%, enquanto para os iniciantes em diálise peritoneal foram de 77\%, 61,6\% e 57,7\% (Figura 1). Para todos os anos, a sobrevida de diálise peritoneal foi estatisticamente diferente da sobrevida em hemodiálise $(\mathrm{p}<0,05)$. Nos primeiros meses observa-se, uma superposição das curvas de sobrevida, o que poderia ser indicativo de falha na proporcionalidade dos riscos, porém, na análise de resíduos log minus log, das modalidades de tratamento, não houve o rompimento desta premissa.

$\mathrm{Na}$ análise do risco estimado de morte com base nas variáveis independentes o modelo final (Tabela 2) indicou as seguintes características associadas a maior risco de falha de tratamento ao final de 3 anos de acompanhamento: ser do sexo feminino, ter idade superior a 55 anos no momento do inicio do tratamento, ter diagnóstico primário de diabetes mellitus, encontrar-se em modalidade de diálise peritoneal e não residir na região sudeste. Residir em cidades com melhores índices de IDH proporcionou menor risco para o óbito.

A análise de resíduos foi testada de acordo com gráfico $\log$ minus $\log$ e mostrou que não houve violação da premissa de risco proporcional no modelo final, uma vez que o efeito de todas as variáveis incluídas se comportou de forma proporcional no tempo analisado.

Avaliando a razão de risco estimado entre as modalidades de diálise para grupos de pacientes segundo as variáveis sexo, faixa etária (superior e inferior a 55 anos) e ter diabetes mellitus como causa básica da insuficiência renal crônica terminal, observa-se que para o primeiro ano só há diferença para o grupo composto por mulheres com idade inferior a 55 anos, que têm menor risco de morte quando realizam diálise peritoneal no primeiro ano (Tabela 3). No segundo ano de tratamento, as mulheres com idade inferior a 55 anos perdem este efeito protetor e aparece uma diferença significativa entre as modalidades, 
Características demográficas e clínicas dos pacientes incidentes submetidos à terapia renal substitutiva segundo modalidade inicial de tratamento, Brasil, 2002-2003.

\begin{tabular}{|c|c|c|c|c|c|c|}
\hline \multirow[t]{2}{*}{ Variável } & \multicolumn{2}{|c|}{ Hemodiálise } & \multicolumn{2}{|c|}{$\begin{array}{c}\text { Diálise } \\
\text { peritoneal }\end{array}$} & \multicolumn{2}{|c|}{ Total } \\
\hline & n & $\%$ & $\mathbf{n}$ & $\%$ & $\mathbf{n}$ & $\%$ \\
\hline Total & 28.062 & 100 & 3.236 & 100 & 31.298 & 100 \\
\hline \multicolumn{7}{|l|}{ Sexo } \\
\hline Masculino & 16.157 & 58 & 1.577 & 49 & 17.734 & 57 * \\
\hline Feminino & 11.909 & 42 & 1.659 & 51 & 13.564 & 43 * \\
\hline \multicolumn{7}{|l|}{ Idade à entrada em terapias renais substitutivas } \\
\hline Média & \multicolumn{2}{|c|}{53,3} & \multicolumn{2}{|c|}{59,5} & \multicolumn{2}{|c|}{54,0 * } \\
\hline Desvio padrão & \multicolumn{2}{|c|}{15,8} & \multicolumn{2}{|c|}{16,0} & \multicolumn{2}{|c|}{16,0} \\
\hline \multicolumn{7}{|l|}{ Faixa etária à entrada em terapias renais substitutivas (anos) } \\
\hline$\leq 55$ & 14.589 & 52 & 1.154 & 36 & 15.743 & 50 * \\
\hline$>55$ & 13.473 & 48 & 2.082 & 64 & 15.555 & 50 * \\
\hline \multicolumn{7}{|l|}{ Causa de insuficiência renal crônica terminal no $1 \underline{0}$ registro } \\
\hline Diabete mellitus & 4.292 & 15 & 633 & 20 & 4.925 & 16 * \\
\hline Hipertensão - doenças cardiovasculares & 6.600 & 24 & 662 & 20 & 7.262 & 23 * \\
\hline Glomerulonefrites & 2.600 & 9 & 193 & 6 & 2.793 & 9 * \\
\hline Indeterminada/Outras doenças & 14.570 & 52 & 1.748 & 54 & 16.318 & 52 * \\
\hline \multicolumn{7}{|l|}{ Região de residência no $1 \underline{0}$ registro } \\
\hline Sudeste & 13.913 & 50 & 1.956 & 60 & 15.869 & 51 * \\
\hline Nordeste & 6.786 & 24 & 529 & 16 & 7.315 & 23 * \\
\hline Centro-oeste & 1.454 & 5 & 143 & 4 & 1.597 & 5 \\
\hline Sul & 4.467 & 16 & 481 & 15 & 4.948 & 16 \\
\hline Norte & 1.442 & 5 & 127 & 4 & 1.569 & 5 * \\
\hline \multicolumn{7}{|l|}{ IDH para o ano de 2000} \\
\hline Média & \multicolumn{2}{|c|}{0,78} & \multicolumn{2}{|c|}{0,79} & \multicolumn{2}{|c|}{0,78 * } \\
\hline \multicolumn{7}{|l|}{ Ano de início de tratamento } \\
\hline 2002 & 13.769 & 49 & 1.566 & 48 & 15.335 & 49 \\
\hline 2003 & 14.293 & 51 & 1.670 & 52 & 15.963 & 51 \\
\hline \multicolumn{7}{|l|}{ Sequência de tratamento durante o período observado } \\
\hline Permaneceu na mesma modalidade inicial & 25.330 & 90 & 2.621 & 81 & 27.951 & 89 * \\
\hline Transitou entre as modalidades de diálise & 1.127 & 4 & 487 & 15 & 1.614 & 5 * \\
\hline Realizou transplante renal & 1.605 & 6 & 128 & 4 & 1.733 & 6 * \\
\hline \multicolumn{7}{|l|}{ Óbitos durante o período de observação } \\
\hline Total & 9.398 & 33 & 1.368 & 42 & 10.766 & 34 * \\
\hline \multicolumn{7}{|l|}{ Causas de óbito } \\
\hline Diabete mellitus não especificado - com complicações renais & 1.334 & 14 & 247 & 18 & 1.581 & 15 * \\
\hline Insuficiência renal crônica não especificada & 1.302 & 14 & 193 & 14 & 1.495 & 14 * \\
\hline Doença renal hipertensiva com insuficiência renal & 708 & 8 & 77 & 6 & 785 & 7 \\
\hline Outras causas de óbito & 6.054 & 64 & 851 & 62 & 6.905 & 64 * \\
\hline
\end{tabular}

Fonte: Base Nacional de Dados em Terapias Renais Substitutivas.

* $\mathrm{p}<0,05$.

IDH: Índice de Desenvolvimento Humano.

com piores escores para diálise peritoneal, para mulheres com idade superior a 55 anos, e para os homens não diabéticos com idade inferior a 55 anos. No terceiro ano a idade apresenta-se como fator de risco para diálise peritoneal, independente do sexo ou da causa básica da insuficiência renal crônica terminal. Observa-se ademais um risco significativamente aumentado para 


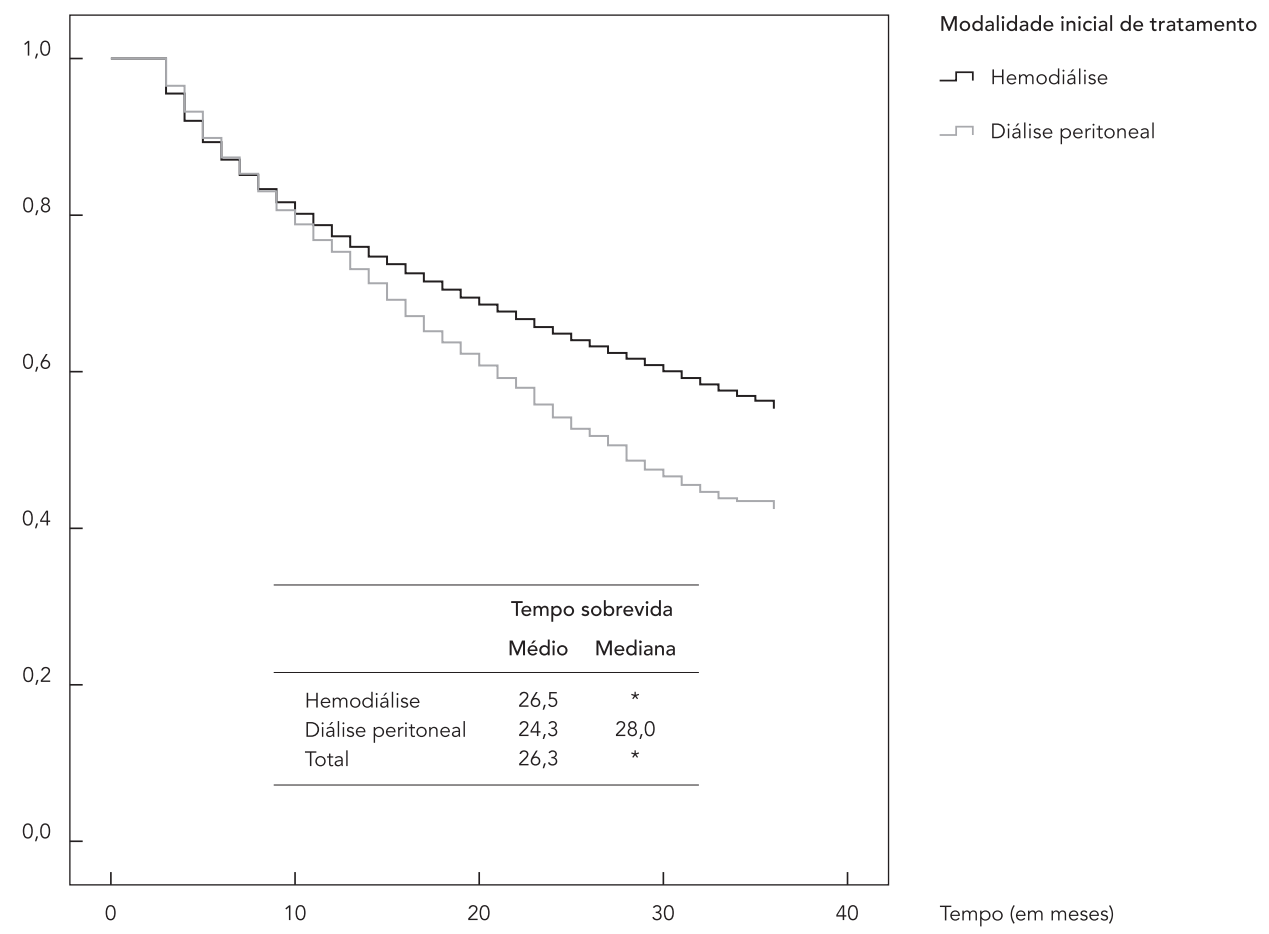

* No tempo avaliado, houve menos de $50 \%$ de óbitos.

Fonte: Base Nacional de Dados em Terapias Renais Substitutivas.

diálise peritoneal em homens, não diabéticos e com idade inferior a 55 anos.

\section{Discussão}

Esse estudo apresenta as primeiras análises acerca da sobrevida de pacientes em tratamento dialítico para o Brasil, avaliando a partir da modalidade inicial de tratamento. Quando considerados os 3 anos de acompanhamento, observou-se que pacientes que iniciaram o tratamento em diálise peritoneal apresentaram piores resultados de sobrevida, em relação àqueles que iniciaram em hemodiálise. Apresentaram tempo mediano de sobrevida de 28 meses, enquanto $50 \%$ dos pacientes que iniciam na modalidade hemodiálise, no período de acompanhamento, não chegaram a óbito. Os fatores que contribuíram para o maior risco de morte dos pacientes foram: iniciar em diálise peritoneal, ser do sexo feminino, ter idade superior a 55 anos ao início do tratamento, ter diabetes mellitus como causa básica da in- suficiência renal crônica terminal, residir fora da região sudeste, e iniciar tratamento em cidades com piores índices de IDH.

Analisando as diferenças de sobrevida entre as modalidades de diálise em pacientes do MedCare (Estados Unidos), entre os anos de 1995 e 2000, Vonesh et. al. 17 observaram que em 42 meses de acompanhamento, o tempo mediano de sobrevida de pacientes em hemodiálise foi de 35,1 meses, enquanto que para os em diálise peritoneal foi de 33,8 meses. van Biesen et al. 16 avaliando pacientes americanos, com início de tratamento dialítico entre 1979 e 1996 e com no mínimo 3 meses na modalidade inicial, encontraram tempos medianos de sobrevida de 51 meses para os pacientes com início em hemodiálise e 42 para os iniciantes em diálise peritoneal.

Recorrentemente, são descritos como fatores que influenciam a sobrevida dos pacientes em diálise o incremento da idade e a existência de diabetes mellitus 10,12,13,17. Inrid et al. 22, avaliando a sobrevida em 2 anos de 12.568 pacientes na fila de espera para transplante renal dos Estados 
Razão de riscos estimada (HR) e intervalo de 95\% de confiança (IC95\%) segundo análise proporcional de Cox para todo o tempo de sobrevida do paciente, Brasil, 2002-2003.

Análise sobrevida total para os 3 anos de acompanhamento

\begin{tabular}{|c|c|c|c|}
\hline \multirow[b]{2}{*}{ Variáveis do modelo } & \multicolumn{2}{|c|}{ Análise univariada } & \multirow{2}{*}{$\begin{array}{c}\text { Análise multivariada } \\
\text { HR (IC95\%) }\end{array}$} \\
\hline & -2 Log Likelihood & HR (IC95\%) & \\
\hline Modalidade inicial (diálise peritoneal/hemodiálise) & $214.527,06$ & $1,32(1,24-1,39)$ * & $1,17(1,1-1,23)$ * \\
\hline Sexo (feminino/masculino) & $214.591,69$ & $1,09(1,05-1,13)$ * & $1,09(1,05-1,14)$ * \\
\hline Idade (> 55 anos/< 55 anos) & $212.692,01$ & $2,38(2,29-2,48)$ * & $2,33(2,24-2,43)$ * \\
\hline Causa de IRCT (diabete mellitus/sem diabete) & $214.426,79$ & $1,4(1,34-1,47)$ & $1,22(1,16-1,28)$ * \\
\hline Região de residência (demais/Sudeste) & $214.566,63$ & $1,14(1,1-1,18)$ * & $1,15(1,11-1,2)$ * \\
\hline IDH (contínua) & $214.584,10$ & $0,74(0,57-0,96)$ * & $0,7(0,53-0,93)$ * \\
\hline
\end{tabular}

Fonte: Base Nacional de Dados em Terapias Renais Substitutivas.

* $\mathrm{p}<0,05$.

IDH: Índice de Desenvolvimento Humano; IRCT: insuficiência renal crônica terminal.

Unidos, detectaram aumento de $26 \%$ na chance de morrer ao incremento de 10 anos na idade inicial de tratamento; de $57 \%$ para aqueles que tinham a diabetes mellitus como causa da insuficiência renal crônica terminal, ajustado por outras características demográficas, de comorbidades e de testes laboratoriais. Lee et al. 23 , em estudo com 1.347 pacientes entre 1991-2005, em um hospital chinês, identificaram como fatores independentes de risco para morte, idade ( $\mathrm{HR}=$ $1,03$ ), ser do sexo masculino ( $H R=2,85)$, ter diabetes mellitus como causa da doença renal ( $\mathrm{HR}=$ $1,49)$ além dos níveis de colesterol, hemoglobina, creatinina e albumina.

Em nosso estudo, o sexo feminino esteve associado ao maior risco de morte no tratamento dialítico ( $\mathrm{HR}=1,09)$. Villar et al. 24 , avaliando 3.025 pacientes incidentes, na região francesa de Rhône-Alpes, ao comparar a mortalidade dos indivíduos em diálise com a população francesa observaram um maior risco de morte para mulheres. Vonesh et al. 5, avaliando 398.940 pacientes em terapia renal substitutivas, observaram um maior risco de morte para o sexo feminino no grupo de pacientes com início de tratamento em hemodiálise, ajustado por idade, raça, causa da doença renal, comorbidades, taxas do ritmo de filtração glomelurar, de albumina e de hemoglobina. Villar et al. 24, avaliando 28.584 indivíduos em terapia renal substitutiva na Austrália e Nova Zelândia, observaram que pertencer ao sexo feminino está associado a um risco maior de morte em pacientes diabéticos tipo $2 \mathrm{com}$ idade acima de 60 anos (HR $=1,19)$. Dentre as explicações para um maior risco da população feminina, encontra-se um efeito da dosagem de diálise (que deveria diferenciar-se por gênero) e a interação entre a menopausa e o risco de doenças cardiovasculares 25 . No Brasil, é recomendável adicionar a estes efeitos as desigualdades de acesso e utilização do sistema de saúde.

Este trabalho apresenta que residir em cidades com melhores índices de IDH, ou seja, com melhores condições socioeconômicas diminui o risco de morte. Sanabria et al. 14 , avaliando o tempo em tratamento dialítico de 923 pacientes colombianos incidentes entre 2001 e 2003, entre os quais $53 \%$ foram tratados com diálise peritoneal, relacionaram como fatores de risco para a sobrevida: ter idade superior a 65 anos (HR = 1,98 ), o diabetes mellitus como causa da insuficiência renal crônica terminal $(\mathrm{HR}=1,76)$ e pior nível socioeconômico $(\mathrm{HR}=2,7)$. No estudo de Sanabria et al. ${ }^{14}$, a maior amplitude de cobertura oferecida pelo Sistema de Previdência Social e de Saúde colombiano diminuiu o risco de morte dos pacientes dialíticos, assim como diminuiu o número de comorbidades apresentadas pelos pacientes. Além das desigualdades regionais brasileiras comumente referenciadas, a diferença do risco pode ser atribuída a problemas de acesso aos serviços que se localizam preferencialmente em municípios pólos 25,26.

Outro fator comumente avaliado e associado ao risco de morte é a modalidade na qual o paciente inicia o tratamento que, para este estudo, é maior para os indivíduos iniciantes em diálise peritoneal, quando analisada a sobrevida para os 3 anos de tratamento, mesmo que ajustado por outros fatores (idade, sexo, causa da insuficiên- 
Razão de riscos (HR) estimada e intervalo de 95\% de confiança (IC95\%) segundo o modelo de análise proporcional de Cox para todos indivíduos incidentes conforme ano de tratamento, Brasil, 2002-2004.

\begin{tabular}{|c|c|c|c|c|}
\hline \multirow[t]{2}{*}{ Sexo/Grupo etário (anos) } & \multicolumn{2}{|c|}{ Não diabético } & \multicolumn{2}{|c|}{ Diabético } \\
\hline & HR (IC95\%) & Valor de $p$ & HR (IC95\%) & Valor de $p$ \\
\hline \multicolumn{5}{|c|}{ Diálise peritoneal e hemodiálise - sobrevida total } \\
\hline \multicolumn{5}{|c|}{ para o primeiro ano de tratamento } \\
\hline \multicolumn{5}{|l|}{ Homem } \\
\hline$<55$ & $1,17(0,9-1,54)$ & 0,241 & $1,09(0,69-1,7)$ & 0,721 \\
\hline$>55$ & $0,9(0,78-1,03)$ & 0,137 & $1,08(0,82-1,43)$ & 0,597 \\
\hline \multicolumn{5}{|l|}{ Mulher } \\
\hline$<55$ & $0,73(0,57-0,95)$ & 0,017 & $0,46(0,23-0,9)$ & 0,023 \\
\hline$>55$ & $0,99(0,86-1,14)$ & 0,933 & $1,03(0,8-1,31)$ & 0,840 \\
\hline \multicolumn{5}{|c|}{ Diálise peritoneal e hemodiálise Sobrevida total } \\
\hline \multicolumn{5}{|c|}{ para o segundo ano de tratamento } \\
\hline \multicolumn{5}{|l|}{ Homem } \\
\hline$<55$ & $1,34(1,09-1,64)$ & 0,006 & $1,04(0,72-1,51)$ & 0,827 \\
\hline$>55$ & $1,06(0,95-1,18)$ & 0,275 & $1,2(0,96-1,49)$ & 0,103 \\
\hline \multicolumn{5}{|l|}{ Mulher } \\
\hline$<55$ & $0,84(0,69-1,03)$ & 0,098 & $0,89(0,6-1,32)$ & 0,579 \\
\hline$>55$ & $1,17(1,05-1,3)$ & 0,006 & $1,23(1,02-1,49)$ & 0,029 \\
\hline \multirow{2}{*}{\multicolumn{5}{|c|}{$\begin{array}{l}\text { Diálise peritoneal e hemodiálise - Sobrevida total } \\
\text { para os três anos de tratamento }\end{array}$}} \\
\hline & & & & \\
\hline \multicolumn{5}{|l|}{ Homem } \\
\hline$<55$ & $1,34(1,1-1,63)$ & 0,004 & $0,98(0,68-1,4)$ & 0,905 \\
\hline$>55$ & $1,13(1,02-1,25)$ & 0,021 & $1,28(1,04-1,57)$ & 0,019 \\
\hline \multicolumn{5}{|l|}{ Mulher } \\
\hline$<55$ & $0,93(0,78-1,12)$ & 0,467 & $0,92(0,64-1,33)$ & 0,646 \\
\hline$>55$ & $1,17(1,05-1,3)$ & 0,005 & $1,24(1,04-1,49)$ & 0,018 \\
\hline
\end{tabular}

Fonte: Base Nacional de Dados em Terapias Renais Substitutivas.

* $p<0,05$

cia renal crônica terminal, região de residência e IDH do município de residência). A literatura é controversa e apresenta resultados que indicam um risco aumentado para diálise peritoneal principalmente quando associado a outros fatores, como comorbidades, idade e tempo de tratamento 12,13 .

Numa perspectiva temporal, avaliando-se os fatores a cada ano de seguimento, observou-se que iniciar em diálise peritoneal no primeiro ano diminui o risco de morte para mulheres com idade inferior a 55 anos. No segundo ano, iniciar em diálise peritoneal apresentou-se como fator de risco para mulheres maiores de 55 anos. Para os três anos de tratamento, conclui-se que a idade é fator importante para maior risco em diálise peritoneal, independente do sexo e da causa básica da insuficiência renal crônica terminal. Excepcionalmente, homens com idade inferior a 55 anos (e não diabéticos) apresentaram risco aumentado para diálise peritoneal, nos 3 anos de tratamento (HR = 1,34).

Os melhores escores identificados para diálise peritoneal no ano inicial de tratamento, quando ajustado pelas covariáveis, foram descritos por Vonesh et al. 17 que, em revisão de 9 artigos comparando as duas modalidades de diálise, identificaram que o risco estimado de morte para diálise peritoneal versus hemodiálise variava com o tempo em terapia - a diálise peritoneal apresentava uma taxa de mortalidade igual ou inferior durante os 2 primeiros anos e, posteriormente, tendia a ser pior do que a hemodiálise. $\mathrm{O}$ maior tempo em tratamento é um fator de risco substancial para quem realiza a diálise peritoneal, quando comparado àqueles que realizam hemodiálise 13,16,27. Este risco pode ser explicado pela perda progressiva da função residual dos 
rins, desejável para a realização da diálise peritoneal, sobrecarga da membrana peritoneal, gerando complicações associadas, como a hipertensão e peritonites podendo inviabilizar a realização do procedimento 12 .

Segundo van Biesen et al. 16, potencializam-se os resultados de sobrevida dos indivíduos quando observa-se um cuidado integrado (melhor sobrevida ente pacientes que iniciaram em diálise peritoneal e foram transferidos para hemodiálise) quando as melhores contribuições de cada modalidade de tratamento podem ser oferecidas aos pacientes.

Para este estudo, a pouca variação no tempo médio de sobrevida observado entre as abordagens propostas por van Biesen et al. 16 (avaliação do tempo em primeira modalidade, tempo intention to treat e tempo total de tratamento), pode dever-se a alta proporção de pacientes que se mantêm na mesma modalidade de início de tratamento, durante todo o tempo de estudo, ou seja, $90 \%$ dos que iniciam em hemodiálise e $81 \%$ dos que iniciam diálise peritoneal. A contribuição dos que transitam entre modalidades de tratamento, inclusive realizando o transplante renal, pode não ter gerado efeito suficiente para modificar o tempo médio de sobrevida, considerando o desenho observacional do estudo, mas pode ter produzido alguma subestimação da razão de riscos referente ao método de diálise.

Quanto às limitações deste estudo, inicialmente é necessário cautela na interpretação dos resultados uma vez que os registros de dados apresentavam $52 \%$ dos indivíduos sem a identificação da causa básica da insuficiência renal crônica terminal. Entretanto, esta observação condiz com a realidade brasileira no que diz respeito às precárias condições clínicas em que os pacientes se apresentam ao início das terapias renais substitutivas, dificultando o diagnóstico da doença de base ${ }^{28}$. Ademais, trata-se de uma base de dados cuja origem é de ordem administrativa, não sendo originalmente construída para estudos científicos 29. Estudos internacionais realçam informações tais como etnia, renda e situação de comorbidades. A fim de minimizar os efeitos da não observação destas variáveis e, portanto, aumentar a comparabilidade do estudo, usou-se o IDH como estimador das condições socioeconômicas dos municípios de residência dos pacientes. Apesar de potencializar os resultados o IDH do município não pode ser usado como estimador das condições individuais. $\mathrm{O}$ IDH médio para todas as cidades brasileiras, no ano de 2000 foi 0,785 , coincidente com a média das cidades onde ocorreram o primeiro registro de tratamento dialítico da população em estu- do. Por fim é necessário realçar que o tempo de análise, 3 anos, é insuficiente para que se possa afirmar que as diferenças de sobrevida encontradas seguirão o padrão de países desenvolvidos no qual ocorre acentuação das diferenças entre as curvas de sobrevida das duas modalidades de tratamento 16 .

Em estudo anterior realizado no âmbito do projeto terapias renais substitutivas concluiu-se que a alocação dos pacientes não era randômica e que pacientes mais idosos e diabéticos tinham maior probabilidade de iniciar a terapia em diálise peritoneal, o que poderia ser traduzido em piores escores de sobrevida para este grupo de tratamento ${ }^{3}$. Em contraste, para a população dos Estados Unidos, os pacientes em diálise peritoneal são mais jovens e mais saudáveis dos que aqueles que iniciam em hemodiálise 11,15.

Em uma análise conduzida por Davies ${ }^{9}$, revisando 8 estudos e comparando a sobrevida dos pacientes entre as modalidades de diálise, o autor conclui que a sobrevida entre os iniciantes em diálise peritoneal ou hemodiálise é muito semelhante, independente do desenho do estudo e das variáveis consideradas. Observa-se que a diálise peritoneal proporciona a manutenção do estilo de vida e que maiores cuidados devem ser tomados no uso da diálise peritoneal em pacientes idosos diabéticos. Davies ${ }^{9}$ sugere também que há uma superestimação da importância clínica da razão de riscos. Para ele, em um período de 24 meses uma razão de riscos aumentado de 1,15 impacta em apenas em 3-4 meses na sobrevivência. Portanto, questões de qualidade de vida para o paciente devem ser consideradas quando da tomada de decisões no tratamento 9 .

No caso deste estudo, o risco estimado ajustado para os três anos de tratamento foi de HR = 1,17 , em favor da hemodiálise o que significaria uma sobrevida de mais 6 meses para os indivíduos iniciantes nesta modalidade. Acreditamos que este requisito é importante, mas ele depende das técnicas de tratamento aplicadas aos pacientes segundo os fatores de risco específicos de cada grupo. Abre-se, portanto, a necessidade de estudos que forneçam um maior volume de informações clínicas dos pacientes. Assim, mais do que resultados numéricos, é necessário repensar a prática clínica, avaliando a possibilidade de utilizar as modalidades segundo o perfil sóciodemográfico e clínico dos pacientes. Adicionalmente poderia se pensar numa reformulação da política da atenção do portador de doença renal crônica, incentivando a realização das modalidades de forma integrada, por meio de estímulos como formas específicas de remuneração ao tratamento. 


\section{Resumo}

O objetivo deste estudo foi analisar a sobrevida dos que iniciaram tratamento renal substitutivo em hemodiálise e diálise peritoneal no SUS, entre 2002 a 2004. Estudo observacional, prospectivo não concorrente. Utilizou-se a Base Nacional em Terapias Renais Substitutivas resultante de pareamento probabilístico dos sistemas Autorização de Procedimentos de Alta Complexidade/Sistema de Informações Ambulatoriais e Sistema de Informações sobre Mortalidade do SUS. Incluíram-se os admitidos em 2002 e 2003, com 3 meses de tratamento e maiores de 18 anos. Dos 31.298 pacientes, a maioria iniciou em hemodiálise, era do sexo masculino, com média de 54 anos e residentes na Região Sudeste, e em municípios com IDH médio foi de 0,78. Associou-se a maior risco óbito: sexo feminino, idade superior a 55 anos, diagnóstico de diabete mellitus, em diálise peritoneal, não residir na Região Sudeste. Residir em cidades com melhor IDH proporcionou menor risco. Risco ajustado de $H R=1,17$ em favor da hemodiálise. Os resultados sugerem menor sobrevida para os de diálise peritoneal e mais velhos. Portanto, torna-se necessário subsidiar políticas que avaliem melhor a escolha da modalidade, com estudos que aprofundem os achados encontrados.

Insuficiência Renal Crônica; Diálise; Sobrevida

\section{Colaboradores}

D. A. C. Szuster contribuiu substancialmente para a concepção e planejamento, análise e interpretação dos dados; contribuiu significativamente na elaboração do rascunho. W. T. Caiaffa contribuiu substancialmente para a concepção e interpretação dos dados. E. I. G. Andrade contribuiu significativamente na elaboração do rascunho e na revisão crítica do conteúdo; participou da aprovação da versão final do manuscrito. F. A. Acurcio contribuiu significativamente na elaboração do rascunho e na revisão crítica do conteúdo e participou da aprovação da versão final do manuscrito. M. L. Cherchiglia contribuiu substancialmente para a concepção e planejamento, análise e interpretação dos dados; contribuiu significativamente na elaboração do rascunho e na revisão crítica do conteúdo; participou da aprovação da versão final do manuscrito.

\section{Agradecimentos}

Autores agradecem os integrantes do Grupo de Pesquisa em Economia da Saúde da Universidade Federal de Minas Gerais por sua valorosa contribuição desde o desenho metodológico à revisão final.

\section{Referências}

1. Grassmann A, Gioberge S, Moeller S, Brown G. ESRD patients in 2004: global overview of patient numbers, treatment modalities and associated trends. Nephrol Dial Transplant 2005; 20:2587-93.

2. Sesso R, Lopes AA, Thomé FS, Bevilacqua JL, Romão JEJ, Lugon J. Resultados do censo de diálise da SBN, 2007. J Bras Nefrol 2007; 29:197-202.

3. Andrade MV, Junoy JP, Andrade EI, Acurcio FD, Sesso R, Queiroz OV, et al. Allocation of initial modality for renal replacement therapy in Brazil. Clin J Am Soc Nephrol 2010; 5:637-44.

4. Mazzuchi N, Fernández-Cean JM, Carbonell E. Criteria for selection of ESRD treatment modalities. Kidney Int 2000; 57 Suppl 74:S136-43.

5. Vonesh EF, Snyder JJ, Folley RN, Collins AJ. The differential impact of risk factors on mortality in hemodialysis and peritoneal dialysis. Kidney Int 2004; 66:2389-401.
6. Vecchi AF, Dratwa M, Wiedemann ME. Healthcare systems and end-stage renal disease (ESRD) therapies - an international review: costs and reimbusement/funding of ESRD therapies. Nephrol Dial Transplant 1999; 14 Suppl 6:31-41.

7. Just PM, Charro FT, Tschosik EA, Noe LL, Bhattacharyya SK, Riella MC. Reimbursement and economic factors influencing dialysis modality choice around the world. Nephrol Dial Transplant 2008; 23: 2365-73.

8. Cunha CB, Leon ACP, Schramm JMA, Carvalho MS, Souza Junior PRB, Chain R. Tempo até o transplante e sobrevida em pacientes com insuficiência renal crônica no Estado do Rio de Janeiro, Brasil, 1998-2002. Cad Saúde Pública 2007; 23:805-13.

9. Davies, SJ. Comparing outcomes on peritoneal and hemodialysis: a case study in the interpretation of observational studies. Saudi J Kidney Dis Transpl 2007; 18:24-30. 
10. D'Ávila R, Guerra EMM, Rodrigues CIS, Fernandes FA, Cadaval RAM, Almeida, FA. Sobrevida de pacientes renais crônicos em diálise peritoneal e hemodiálise. J Bras Nefrol 1999; 21:13-21.

11. Ganesh SK, Hulbert-Shearon T, Port FK, Eagle K, Stack AG. Mortality differences by dialysis modality among Incident ESRD patients with and without coronary artery disease. J Am Soc Nephrol 2003; 14:415-24.

12. Jaar BG, Coresh J, Plantinga LC, Fink NE, Klag MJ, Levey AS, et al. Comparing the risk for death with peritoneal dialysis and hemodialysis in a national cohort of patients with chronic kidney disease. Ann Intern Med 2005; 143:174-83.

13. McDonald SP, Marshall MR, Johnson DW, Polkinghorne KR. Relationship between dialysis modality and mortality. J Am Soc Nephrol 2009; 20:155-63.

14. Sanabria M, Muñoz J, Trillos C, Hernandez G, Latorre C, Díaz CS, et al. Dialysis outcomes in Colombia (DOC) study: a comparison of patient survival on peritoneal dialysis vs hemodialysis in Colombia. Kidney Int 2008; 73:165-72.

15. Stack AG, Molony DA, Rahman NS, Dosekun A, Murthy B. Impact of dialysis modality on survival of new ESRD patients with congestive heart failure in the United States. Kidney Int 2003; 64:1071-9.

16. van Biesen W, Vanholder RC, Veys N, Dhondt A, Lameire NH. An evaluation of an integrative care approach for end-stage renal disease patients. J Am Soc Nephrol 2000; 11:116-25.

17. Vonesh EF, Snyder JJ, Foley RN, Collins AJ. Mortality studies comparing peritoneal dialysis and hemodialysis: what do they tell us? Kidney Int Suppl 2006; 103:3-11.

18. Sancho LG, Dain S. Análise de custo-efetividade em relação às terapias renais substitutivas: como pensar estudos em relação a essas intervenções no Brasil? Cad Saúde Pública 2008; 24:1279-90.

19. Cherchiglia ML, Guerra Júnior AA, Andrade EIG, Machado CJ, Acúrcio FA, Meira Júnior W, et al. A construção da base de dados nacional em terapia renal substitutiva (TRS) centrada no indivíduo: aplicação do método de linkage determinísticoprobabilístico. Rev Bras Estud Pop 2007; 24:163-7.

20. Queiroz OV, Guerra Júnior AA, Machado CJ, Andrade EIG, Meira Júnio W, Acúrcio FA, et al. A construção da Base Nacional em Terapia Renal Substitutiva (TRS) centrada no indivíduo: relacionamento entre registros de óbitos do subsistema de Autorização de Procedimentos de Alta Complexidade (APAC) e pelo Sistema de Informações de Mortalidade (SIM). Epidemiol Serv Saúde 2009; 18:107-20.
21. Programa das Nações Unidas para o Desenvolvimento. Relatório do desenvolvimento humano 2006. A água para lá da escassez: poder, pobreza e a crise mundial da água. New York: Programa das Nações Unidas para o Desenvolvimento; 2006.

22. Inrig JK, Sun JL, Yang Q, Briley LP, Szczech LA. Mortality by dialysis modality among patients who have end-stage renal disease and are awaiting renal transplantation. Clin J Am Soc Nephrol 2006; 1:774-9.

23. Lee CC, Sun CY, Wu MS. Long-term modality related analysis in incident dialysis patients. Perit Dial Int 2009; 29:182-90

24. Villar E, Remontet L, Labeeuw M, Ecochard R. Effect of age, gender, and diabetes on excess death in end-stage renal failure. J Am Soc Nephrol 2007; 18:2125-34.

25. Vianna SM, Nunes A, Góes G. Atenção de alta complexidade no SUS: desigualdades no acesso e no financiamento. Projeto economia da saúde. Brasília: Ministério da Saúde/Instituto de Pesquisa Econômica Aplicada; 2005.

26. Oliveira AC, Simões RF, Andrade MV. Regionalização dos serviços de média e alta complexidade hospitalar e ambulatorial em Minas Gerais: estrutura corrente versus estrutura planejada. In: Anais do XIII Seminário sobre a Economia Mineira. http:/ / www.cedeplar.ufmg.br/seminarios/semi nario_diamantina/2008/D08A058.pdf (acessado em 15/Mai/2009).

27. Macintyre SFG, Hund K. Do women "over-report" morbidity? Men's and women's responses to structured prompting on a standard question on long standing illness. Soc Sci Med 1999; 48:89-98.

28. Guerra Junior AA, Cesar CC, Cherchiglia ML, Andrade EIG, Queiroz OV, Silva D. Cyclosporine versus tacrolimus in immunosuppressive maintenance regimens in renal transplants in Brazil: survival analysis from 2000 to 2004. Ann Pharmacother 2009; 44:192-201.

29. Szuster DAC, Silva GM, Andrade EIG, Acúrcio FA, Caiaffa WT, Gomes IC, et al. Potencialidades do uso de bancos de dados para informação em saúde: o caso das Terapias Renais Substitutivas (TRS) - morbidade e mortalidade dos pacientes em TRS. Rev Med Minas Gerais 2009; 19:308-16.

Recebido em 13/Mai/2011

Versão final reapresentada em 24/Ago/2011 Aprovado em 15/Set/2011 